\title{
Spin-3/2 to spin-1/2 heavy baryons and pseudoscalar mesons transitions in QCD
}

\author{
T. M. Aliev ${ }^{* \dagger}$, K. Azizi ${ }^{\ddagger}$, M. Savc1 $§$ \\ Physics Department, Middle East Technical University, 06531 Ankara, Turkey \\ $\ddagger$ Physics Division, Faculty of Arts and Sciences, Doğuş University, \\ Acıbadem-Kadıköy, 34722 Istanbul, Turkey
}

\begin{abstract}
The strong coupling constants of light pseudoscalar mesons with spin- $3 / 2$ and spin- $1 / 2$ heavy baryons are calculated in the framework of light cone QCD sum rules. It is shown that each class of transitions among members of the sextet spin- $3 / 2$ to sextet spin $-1 / 2$ baryons and that of the sextet spin $-3 / 2$ to spin $-1 / 2$ anti-triplet baryons is described by only one invariant function. We also estimate the widths of kinematically allowed transitions. Our results on decay widths are in good agreement with the existing experimental data, as well as predictions of other nonperturbative approaches.
\end{abstract}

PACS number(s): 11.55.Hx, 13.75.Gx, 13.75.Jz

\footnotetext{
*e-mail: taliev@metu.edu.tr

†permanent address: Institute of Physics,Baku,Azerbaijan

†e-mail: kazizi@dogus.edu.tr

§e-mail: savci@metu.edu.tr
} 


\section{Introduction}

Theoretical and experimental studies of the flavored hadrons are among the most promising areas in particle physics. From theoretical point of view, this can be explained by the fact that the heavy flavored baryons provide a rich laboratory to study predictions of the heavy quark effective theory. On the other hand, these baryons have many weak and strong decay channels and therefore measurement of these channels can give essential information about the quark structure of heavy baryons. During the last decade, highly exciting experimental results have been obtained on the spectroscopy of heavy hadrons. All ground states of heavy hadrons with $c$ quark have been observed [1]. The new states of heavy baryons are also discovered in BaBar, BELLE, CDF and D $\varnothing$ Collaborations. The operation of LHC will open a new window for more detailed investigation of these new baryons [2].

At present, we have experimental information on the strong one-pion decays for the $\Sigma_{c}$ $[3-5], \Sigma_{c}^{*}[4,6]$ and $\Xi_{c}^{*}[7,8]$ baryons. The strong coupling constants of pseudoscalar mesons with heavy baryons are the main unknown parameters of these transitions. Therefore, a reliable estimation of these strong coupling constants in the framework of QCD receives great interest. At hadronic scale, the strong coupling constant $\alpha_{s}\left(Q^{2}\right)$ is large and hence perturbative theory becomes invalid. For this reason, estimation of the coupling constants becomes impossible starting from the fundamental QCD Lagrangian and some nonperturbative methods are needed. Among many nonperturbative approaches, the QCD sum rule [9] is one of the most powerful method in studying the properties of hadrons. The main advantage of this method is that, it is based on fundamental QCD Lagrangian. In the present work, we estimate the strong coupling constants of pseudoscalar mesons in the transitions of spin-3/2 to spin-1/2 heavy baryons within light cone QCD sum rules method (for more about this method, see [10]). Note that the strong coupling constants of pseudoscalar and vector mesons with heavy baryons in the spin-1/2 to spin-1/2 transitions are studied in [11] and [12].

The rest of the paper is organized as follows. In section 2 , the light cone sum rules for the coupling constants of pseudoscalar mesons with heavy baryons in spin- $3 / 2$ to spin $-1 / 2$ transitions are calculated. In section 3 , the numerical analysis of the obtained sum rules is performed and a comparison of our results with the predictions of other approaches as well as existing experimental results is made.

\section{Light cone QCD sum rules for the pseudoscalar mesons with heavy baryons in spin- $3 / 2$ to spin- $1 / 2$ transi- tions}

In this section, the strong coupling constants of light pseudoscalar mesons with heavy baryons in spin-3/2 to spin-1/2 transitions are calculated. Before making an attempt in estimating these coupling constants, few words about $S U(3)_{f}$ classification of heavy baryons are in order. Heavy baryons with a single heavy quark and two light quarks can be decomposed into two multiplets, namely, sextet $6_{F}$ and anti-triplet $\overline{3}_{F}$ due to the symmetry property of flavor and color structures of these baryons. This observation leads to the result 
of total spin $J^{P}=(3 / 2)^{+}$or $(1 / 2)^{+}$for $6_{F}$ and $J^{P}=(1 / 2)^{+}$for $\overline{3}_{F}$. In the present work, we consider $J=3 / 2$ states in $6_{F}$ and investigate sextet to sextet ( $\mathrm{S}^{*} \mathrm{SP}$ ) and sextet to anti-triplet $\left(\mathrm{S}^{*} \mathrm{AP}\right)$ transitions with the participation of light pseudoscalar mesons, where $S^{*}, S$ and $A$ stand for sextet with spin-3/2, sextet with spin- $1 / 2$ and anti-triplet spin-1/2 states, respectively.

We now pay our attention to the calculation of the strong coupling constants of pseudoscalar mesons with heavy baryons in spin- $3 / 2$ to spin- $-1 / 2$ transitions. To derive the light cone sum rules for $S^{*} S P$ and $S^{*} A P$ transitions we consider the following general correlation function:

$$
\Pi_{\mu}^{(i)}=i \int d^{4} x e^{i p x}\left\langle P(q)\left|\eta^{(i)}(x) \bar{\eta}_{\mu}(0)\right| 0\right\rangle,
$$

where $\eta^{(i)}(x)$ are the interpolating currents of the heavy baryons with spin- $1 / 2$ in sextet $(i=1)$ and anti-triplet $(i=2)$ representation and $\bar{\eta}_{\mu}$ is the interpolating current for the sextet $J^{P}=3^{+} / 2$ states. The correlation function (1) can be calculated in terms of hadrons (phenomenological part) and in terms of quark-gluon degrees of freedom in deep Euclidean region, i.e., when $p^{2} \rightarrow-\infty$. Equating then these representations of the correlation function using the dispersion relation, we get the sum rules for strong coupling constants of light pseudoscalar mesons with heavy baryons.

We proceed by calculating the phenomenological part of the correlation function. The expression for the phenomenological part is obtained by saturating it with the full set of hadrons carrying the same quantum numbers as the corresponding interpolating current. Isolating the contributions of the ground state baryons, one can easily obtain

$$
\Pi_{\mu}=\frac{\left.\left\langle 0\left|\eta^{(i)}(x)\right| B_{(} p\right)\right\rangle\left\langle B(p) P(q) \mid B^{*}(p+q)\right\rangle\left\langle B^{*}(p+q)\left|\bar{\eta}_{\mu}(0)\right| 0\right\rangle}{\left(p^{2}-m_{2}^{2}\right)\left[(p+q)^{2}-m_{1}^{2}\right]}+\cdots,
$$

where $m_{1}$ and $m_{2}$ are the masses of the initial and final heavy baryons, $p+q$ and $p$ represent their four-momentum, respectively, and dots represent contributions coming from higher states and continuum. It follows from Eq. (2) that in obtaining the phenomenological part of the correlation function, the matrix elements, $\left.\left\langle 0\left|\eta^{(i)}(x)\right| B_{(} p\right)\right\rangle,\langle B(p) P(q) \mid B(p+q)\rangle$ and $\left\langle B(p+q)\left|\bar{\eta}_{\mu}(0)\right| 0\right\rangle$ are needed. These matrix elements are determined as follows:

$$
\begin{aligned}
\langle 0|\eta| B(p)\rangle & =\lambda_{2}^{(i)} u(p), \\
\left\langle B^{*}(p+q)\left|\eta_{\mu}\right| 0\right\rangle & =\lambda_{1} \bar{u}_{\mu}(p+q), \\
\left\langle P(q) B(p) \mid B^{*}(p+q)\right\rangle & =g_{B^{*} B P} \bar{u}(p) u_{\alpha}(p+q) q^{\alpha},
\end{aligned}
$$

where $\lambda_{2}$ and $\lambda_{1}$ are the residues of spin-1/2 and spin-3/2 heavy baryons, respectively, $g$ is the coupling constant of heavy baryons with pseudoscalar mesons, and $u_{\mu}$ is the RaritaSchwinger spinor. Using Eqs. (3) and (2) and performing summation over spins of spin-1/2 and spin- $3 / 2$ baryons,

$$
\begin{aligned}
\sum_{s} u(p, s) \bar{u}(p, s) & =\left(\not p+m_{2}\right) \\
\sum_{s} u_{\alpha}(p+q, s) \bar{u}_{\mu}(p+q, s) & =-\left(\not p+\not p+m_{1}\right)\left\{g_{\alpha \mu}-\frac{\gamma_{\alpha} \gamma_{\mu}}{3}-\frac{2(p+q)_{\alpha}(p+q)_{\mu}}{3 m_{1}^{2}}\right. \\
& \left.+\frac{(p+q)_{\alpha} \gamma_{\mu}-(p+q)_{\mu} \gamma_{\alpha}}{3 m_{1}}\right\},
\end{aligned}
$$


in principle, one can obtain the expression for the phenomenological part of the correlation function. But at this point appear two unpleasant problems: a) The spin-1/2 baryons also contribute to the matrix element $\left\langle 0\left|\eta_{\mu}\right| B(p, 3 / 2)\right\rangle$ of spin- $3 / 2$ baryons (see also [11]). Indeed,

$$
\left\langle 0\left|\eta_{\mu}\right| B(p, 1 / 2)\right\rangle=A\left(\gamma_{\mu}-\frac{4}{m_{2}} p_{\mu}\right) u(p),
$$

hence, the current $\eta_{\mu}$ couples to both spin-3/2 and spin- $-1 / 2$ states. Using Eqs. (2), (4) and (5), one can see that the unwanted contributions coming from spin- $1 / 2$ states contain structures proportional to $\gamma_{\mu}$ at the far right end or $(p+q)_{\mu}$. b) The second problem is related to the fact that the structures which appear in the phenomenological part of the correlation function are not all independent. Both these problems can be removed by ordering the Dirac matrices in a specific form. In this work, the Dirac matrices are ordered in the form $\not p p \gamma_{\mu}$ and the coefficient of the structure $\not q q_{\mu}$ is chosen in order to calculate the aforementioned strong coupling constant, which is free of the spin- $1 / 2$ contributions. Using the ordering procedure, we get the following representation for the coefficient of the selected structure in the phenomenological part:

$$
\Pi_{\mu}^{(i)}=\frac{g \lambda_{1} \lambda_{2}^{(i)} m_{2}}{\left[m_{1}^{2}-(p+q)^{2}\right]\left(m_{2}^{2}-p^{2}\right)}+\text { other structures. }
$$

In order to obtain sum rules for the coupling constant appearing in Eq. (6), we need to calculate the correlation function also from the QCD side. Before calculating it, we shall first find the relations among the correlation functions corresponding to different transition channels. In more concrete words, we shall find the relations among the coefficient functions of the structure $\not q q_{\mu}$ for different transition channels. For this purpose we follow an approach whose main ingredients are presented in [14-17].

In obtaining the relations among the correlation functions describing various spin- $3 / 2$ to spin-1/2 heavy baryon transitions, as well as, in obtaining the theoretical part of QCD sum rules, the forms of the interpolating currents are needed. In constructing the interpolating currents, we will use the fact that the interpolating currents for the particles in sextet (anti-triplet) representations should be symmetric (antisymmetric) with respect to the light quarks. Using this fact, the interpolating current for baryons in sextet representation with $J=3 / 2$ can be written as:

$$
\eta_{\mu}=A \epsilon^{a b c}\left\{\left(q_{1}^{a} C \gamma_{\mu} q_{2}^{b}\right) Q^{c}+\left(q_{2}^{a} C \gamma_{\mu} Q^{b}\right) q_{1}^{c}+\left(Q^{a} C \gamma_{\mu} q_{1}^{b}\right) q_{2}^{c}\right\}
$$

where $A$ is the normalization factor, $a, b$ and $c$ are the color indices. In Table 1 , we present the values of $A$ and light quark content of heavy spin-3/2 baryons. The general form of the interpolating currents for the heavy spin-1/2 sextet and antitriplet baryons can be written as ( for example see [18]):

$$
\begin{aligned}
\eta_{Q}^{(s)} & =-\frac{1}{\sqrt{2}} \epsilon^{a b c}\left\{\left(q_{1}^{a T} C Q^{b}\right) \gamma_{5} q_{2}^{c}+\beta\left(q_{1}^{a T} C \gamma_{5} Q^{b}\right) q_{2}^{c}-\left[\left(Q^{a T} C q_{2}^{b}\right) \gamma_{5} q_{1}^{c}+\beta\left(Q^{a T} C \gamma_{5} q_{2}^{b}\right) q_{1}^{c}\right]\right\}, \\
\eta_{Q}^{(a)} & =\frac{1}{\sqrt{6}} \epsilon^{a b c}\left\{2\left(q_{1}^{a T} C q_{2}^{b}\right) \gamma_{5} Q^{c}+2 \beta\left(q_{1}^{a T} C \gamma_{5} q_{2}^{b}\right) Q^{c}+\left(q_{1}^{a T} C Q^{b}\right) \gamma_{5} q_{2}^{c}+\beta\left(q_{1}^{a T} C \gamma_{5} Q^{b}\right) q_{2}^{c}\right. \\
& \left.+\left(Q^{a T} C q_{2}^{b}\right) \gamma_{5} q_{1}^{c}+\beta\left(Q^{a T} C \gamma_{5} q_{2}^{b}\right) q_{1}^{c}\right\},
\end{aligned}
$$




\begin{tabular}{|c|c|c|c|c|c|c|}
\hline \hline & $\Sigma_{b(c)}^{*+(++)}$ & $\Sigma_{b(c)}^{* 0(+)}$ & $\Sigma_{b(c)}^{*-(0)}$ & $\Xi_{b(c)}^{* 0(+)}$ & $\Xi_{b(c)}^{*-(0)}$ & $\Omega_{b(c)}^{*-(0)}$ \\
\hline$q_{1}$ & $u$ & $u$ & $d$ & $u$ & $d$ & $s$ \\
$q_{2}$ & $u$ & $d$ & $d$ & $s$ & $s$ & $s$ \\
$A$ & $\sqrt{1 / 3}$ & $\sqrt{2 / 3}$ & $\sqrt{1 / 3}$ & $\sqrt{2 / 3}$ & $\sqrt{2 / 3}$ & $\sqrt{1 / 3}$ \\
\hline \hline
\end{tabular}

Table 1: The light quark content $q_{1}$ and $q_{2}$ for the sextet baryons with spin- $3 / 2$

where $\beta$ is an arbitrary constant and $\beta=-1$ corresponds to the Ioffe current and superscripts $s$ and $a$ stand for symmetric and antisymmetric spin- $1 / 2$ currents, respectively. The light quark content of the heavy baryons with spin- $-1 / 2$ in the sextet and anti-triplet representations are given in Table 2 .

\begin{tabular}{|c|c|c|c|c|c|c|c|c|c|}
\hline \hline & $\Sigma_{b(c)}^{+(++)}$ & $\Sigma_{b(c)}^{0(+)}$ & $\Sigma_{b(c)}^{-(0)}$ & $\Xi_{b(c)}^{-(0)^{\prime}}$ & $\Xi_{b(c)}^{0(+)^{\prime}}$ & $\Omega_{b(c)}^{-(0)}$ & $\Lambda_{b(c)}^{0(+)}$ & $\Xi_{b(c)}^{-(0)}$ & $\Xi_{b(c)}^{0(+)}$ \\
\hline$q_{1}$ & $u$ & $u$ & $d$ & $d$ & $u$ & $s$ & $u$ & $d$ & $u$ \\
$q_{2}$ & $u$ & $d$ & $d$ & $s$ & $s$ & $s$ & $d$ & $s$ & $s$ \\
\hline \hline
\end{tabular}

Table 2: The light quark content $q_{1}$ and $q_{2}$ for the sextet and anti-triplet baryons with spin- $1 / 2$

After introducing the explicit expressions for the interpolating currents, we are ready now to obtain the relations among the correlation functions that describe different transitions. It should be noted here that the relations which are presented below are independent of the choice of structures, while the expressions of the correlation functions are all structure dependent.

In order to obtain the relations among the correlation functions responsible for different transitions, we consider the $\Sigma_{b}^{* 0} \rightarrow \Sigma_{b}^{0} \pi^{0}$ and $\Sigma^{* 0} \rightarrow \Lambda \pi^{0}$ transitions which describe sextet spin $-3 / 2$ to sextet spin- $1 / 2$ and sextet spin $-3 / 2$ to anti-triplet spin $-1 / 2$ transitions, respectively. These invariant functions can be written in the general form as

$$
\begin{aligned}
\Pi^{\Sigma_{b}^{* 0} \rightarrow \Sigma_{b}^{0} \pi^{0}} & =g_{\pi^{0} \bar{u} u} \Pi_{1}^{(1)}(u, d, b)+g_{\pi^{0} \bar{d} d} \Pi_{1}^{(1)^{\prime}}(u, d, b)+g_{\pi^{0} \bar{b} b} \Pi_{2}^{(1)^{\prime}}(u, d, b), \\
\Pi^{\Sigma^{* 0} \rightarrow \Lambda \pi^{0}} & =g_{\pi^{0} \bar{u} u} \Pi_{1}^{(2)}(u, d, b)+g_{\pi^{0} \bar{d} d} \Pi_{1}^{(2)^{\prime}}(u, d, b)+g_{\pi^{0} \bar{b} b} \Pi_{2}^{(2)^{\prime}}(u, d, b),
\end{aligned}
$$

where superscripts (1) and (2) correspond to $\mathrm{S}^{*} \mathrm{SP}$ and $\mathrm{S}^{*} \mathrm{AP}$ transitions, respectively. The interpolating current for $\pi^{0}$ is written as:

$$
J_{\pi^{0}}=\sum g_{\pi^{0} \bar{q} q} \bar{q} \gamma_{5} q=\frac{1}{\sqrt{2}}\left(\bar{u} \gamma_{5} u-\bar{d} \gamma_{5} d\right) .
$$

It follows from this expression that,

$$
g_{\pi^{0} \bar{u} u}=-g_{\pi^{0} \bar{d} d}=\frac{1}{\sqrt{2}}, \quad g_{\pi^{0} \bar{b} b}=0 .
$$


The invariant functions $\Pi_{1}^{(i)}(u, d, b), \Pi_{1}^{(i)^{\prime}}(u, d, b)$, and $\Pi_{2}^{(i)}(u, d, b)$ describe the radiation of $\pi^{0}$ from $u, d$ and $b$ quarks, respectively, and they are formally defined in the following way:

$$
\begin{aligned}
\Pi_{1}^{(i)}(u, d, b) & =\left\langle\bar{u} u\left|\Sigma_{b}^{* 0} \Sigma_{b}^{0}\left(\Lambda_{b}\right)\right| 0\right\rangle, \\
\Pi_{1}^{(i)^{\prime}}(u, d, b) & =\left\langle\bar{d} d\left|\Sigma_{b}^{* 0} \Sigma_{b}^{0}\left(\Lambda_{b}\right)\right| 0\right\rangle, \\
\Pi_{2}^{(i)}(u, d, b) & =\left\langle\bar{b} b\left|\Sigma_{b}^{* 0} \Sigma_{b}^{0}\left(\Lambda_{b}\right)\right| 0\right\rangle .
\end{aligned}
$$

Remembering the fact that the interpolating currents for sextet spin-3/2 and sextet spin- $1 / 2$ baryons are symmetric with respect to the exchange of light quarks, while the interpolating currents for spin- $1 / 2$ anti-triplet baryons are antisymmetric, we can write,

$$
\begin{aligned}
& \Pi_{1}^{(1)^{\prime}}(u, d, b)=\Pi_{1}^{(1)}(d, u, b), \\
& \Pi_{1}^{(2)^{\prime}}(u, d, b)=-\Pi_{1}^{(2)}(d, u, b) .
\end{aligned}
$$

Using these relations and Eqs. (9) and (11), we get,

$$
\Pi^{\Sigma_{b}^{* 0} \rightarrow \Sigma_{b}^{0} \pi^{0}\left(\Sigma^{* 0} \rightarrow \Lambda \pi^{0}\right)}=\frac{1}{\sqrt{2}}\left[\Pi_{1}^{(i)}(u, d, b) \mp \Pi_{1}^{(i)}(d, u, b)\right]
$$

where $i=1(i=2)$ and upper (lower) sign describes $\mathrm{S}^{*} \mathrm{SP}$ (S*AP) transition.

The invariant function responsible for the $\Xi_{b}^{* 0} \rightarrow \Xi_{b}^{0^{\prime}} \pi^{0}$ and $\Xi_{b}^{* 0} \rightarrow \Xi_{b}^{0} \pi^{0}$ transitions can be obtained from the $\Sigma_{b}^{* 0} \rightarrow \Sigma_{b}^{0} \pi^{0}$ and $\Sigma_{b}^{* 0} \rightarrow \Lambda_{b}^{0} \pi^{0}$ channels by noting that the interpolating currents for $\Xi_{b}^{* 0}, \Xi_{b}^{0^{\prime}}$ and $\Xi_{b}^{0}$ can be obtained from the one for $\Sigma_{b}^{* 0}, \Sigma_{b}^{0}$ and $\Lambda_{b}^{0}$ by making the replacement $d \rightarrow s$, and taking into account the fact that $g_{\pi^{0} \bar{s} s}=0$. As a result, we get,

$$
\Pi_{b}^{\Xi_{b}^{* 0} \rightarrow \Xi_{b}^{0^{\prime}} \pi^{0}\left(\Xi_{b}^{* 0} \rightarrow \Xi_{b}^{0} \pi^{0}\right)}=\frac{1}{\sqrt{2}} \Pi_{1}^{(i)}(u, s, b) .
$$

The invariant functions corresponding to $\Xi_{b}^{*-} \rightarrow \Xi_{b}^{-{ }^{\prime}} \pi^{0}$ and $\Xi_{b}^{*-} \rightarrow \Xi_{b}^{-} \pi^{0}$ transitions can be obtained from the $\Xi_{b}^{* 0} \rightarrow \Xi_{b}^{0^{\prime}} \pi^{0}$ and $\Xi_{b}^{* 0} \rightarrow \Xi_{b}^{0} \pi^{0}$ channels with the help of the replacement $u \rightarrow d$, as a result of which we get,

$$
\Pi_{b}^{\Xi_{b}^{*-} \rightarrow \Xi_{b}^{-{ }^{\prime}} \pi^{0}\left(\Xi_{b}^{*-} \rightarrow \Xi_{b}^{-} \pi^{0}\right)}=-\frac{1}{\sqrt{2}} \Pi_{1}^{(i)}(d, s, b)
$$

Calculation of the coupling constants of the sextet spin-3/2 to sextet and anti-triplet spin-1/2 transitions with other pseudoscalar mesons can be done in a similar way as for the $\pi^{0}$ meson. Note that in our calculations, the mixing between $\eta$ and $\eta^{\prime}$ mesons is neglected and the interpolating current of $\eta$ meson has the following form:

$$
J_{\eta}=\frac{1}{\sqrt{6}}\left[\bar{u} \gamma_{5} u+\bar{d} \gamma_{5} d-2 \bar{s} \gamma_{5} s\right]
$$

that gives,

$$
g_{\eta \bar{u} u}=g_{\eta \bar{d} d}=\frac{1}{\sqrt{6}}, \text { and } g_{\eta \bar{s} s}=-\frac{2}{\sqrt{6}} \text {. }
$$


Using this expression let us consider, for example, the $\Sigma_{b}^{* 0} \rightarrow \Sigma_{b}^{0} \eta$ and $\Sigma_{b}^{* 0} \rightarrow \Lambda_{b}^{0} \eta$ transitions. Following the same lines of calculations as in the $\pi^{0}$ meson case, we immediately get,

$$
\Pi_{b}^{\Sigma_{b}^{* 0} \rightarrow \Sigma_{b}^{0} \eta\left(\Sigma_{b}^{* 0} \rightarrow \Lambda_{b}^{0} \eta\right)}=\frac{1}{\sqrt{6}}\left[\Pi_{1}^{(i)}(u, d, b) \pm \Pi_{1}^{(i)}(d, u, b)\right]
$$

The invariant function responsible for the $\Xi_{b}^{* 0} \rightarrow \Xi_{b}^{0^{\prime}} \eta$ and $\Xi_{b}^{* 0} \rightarrow \Xi_{b}^{0} \eta$ transition can be written as:

$$
\begin{aligned}
\Pi_{b}^{* 0} \rightarrow \Xi_{b}^{0^{\prime}} \eta\left(\Xi_{b}^{* 0} \rightarrow \Xi_{b}^{0} \eta\right) & =g_{\eta \bar{u} u} \Pi_{1}^{(i)}(u, s, b)+g_{\eta \bar{s} s} \Pi_{1}^{(i)^{\prime}}(u, s, b)+g_{\eta \bar{b} b} \Pi_{2}^{(i)}(u, s, b) \\
& =\frac{1}{\sqrt{6}}\left[\Pi_{1}^{(i)}(u, s, b)-2 \Pi_{1}^{(i)^{\prime}}(u, s, b)\right] \\
& =\frac{1}{\sqrt{6}}\left[\Pi_{1}^{(i)}(u, s, b) \mp 2 \Pi_{1}^{(i)}(s, u, b)\right] .
\end{aligned}
$$

The relations among invariant functions involving charged pseudoscalar $\pi^{ \pm}$mesons can be obtained from previous results by taking into account the following arguments. For instance, let us consider the $\Sigma_{b}^{*+} \rightarrow \Lambda_{b}^{0} \pi^{+}$transition. In the $\Sigma_{b}^{* 0} \rightarrow \Lambda_{b}^{0} \pi^{0}$ transition, the $u(d)$ quark from $\Sigma_{b}^{* 0}$ and $\Lambda_{b}^{0}$ baryons forms the final $\bar{u} u(\bar{d} d)$ state, and the $d(u)$ and $b$ quarks behave like spectators. In the case of charged $\pi^{+}$meson, the $d$ quark from $\Lambda_{b}^{0}$ and $u$ quark from $\Sigma_{b}^{* 0}$ form the $\bar{u} d$ final state, and the remaining $d(u)$ and $b$ quarks are the spectators. For this reason, one can expect that these two matrix elements should be proportional to each other and explicit calculations show that this indeed is the case. Hence,

$$
\begin{aligned}
\Pi_{b}^{\Sigma_{b}^{*+} \rightarrow \Lambda_{b}^{0} \pi^{+}} & =\left\langle\bar{u} d\left|\Sigma_{b}^{*+} \Lambda_{b}^{0}\right| 0\right\rangle=\sqrt{2}\left\langle\bar{d} d\left|\Sigma_{b}^{* 0} \Lambda_{b}^{0}\right| 0\right\rangle \\
& =-\sqrt{2} \Pi_{1}^{(2)}(d, u, b) .
\end{aligned}
$$

making the replacement $u \leftrightarrow d$ in Eq. (21), we get

$$
\Pi^{\Sigma_{b}^{*-} \rightarrow \Lambda_{b}^{0} \pi^{-}}=\sqrt{2} \Pi_{1}^{(2)}(u, d, b)
$$

All remaining relations among the invariant functions responsible for the spin- $-3 / 2$ and spin-1/2 transitions involving pseudoscalar mesons are presented in the Appendix.

After establishing the relations among the invariant functions, we now proceed by calculating the invariant functions from QCD side in deep Euclidean region $-p^{2} \rightarrow \infty$, $-(p+q)^{2} \rightarrow \infty$, using the operator product expansion (OPE). The main nonperturbative input parameters in the calculation of the theoretical part of the correlation function are the distribution amplitudes (DAs) of the pseudoscalar mesons. These (DAs) of the pseudoscalar mesons are involved in determining the matrix elements of the nonlocal operators between the vacuum and one pseudoscalar meson states, i.e., $\langle p(q)|\bar{q}(x) \Gamma q(0)| 0\rangle$ and $\left\langle p(q)\left|\bar{q}(x) G_{\mu \nu} q(0)\right| 0\right\rangle$, where $\Gamma$ is any Dirac matrix. The DAs of pseudoscalar mesons up to twist -4 accuracy are given in [19].

In the calculation of the theoretical part of the correlation function, we also need to know the expressions of the light and heavy quark propagators. The light quark propagator, in 
presence of an external field, is calculated in [20]:

$$
\begin{aligned}
S_{q}(x)= & \frac{i \not x}{2 \pi^{2} x^{4}}-\frac{m_{q}}{4 \pi^{2} x^{2}}-\frac{\langle\bar{q} q\rangle}{12}\left(1-i \frac{m_{q}}{4} \not x\right)-\frac{x^{2}}{192} m_{0}^{2}\langle\bar{q} q\rangle\left(1-i \frac{m_{q}}{6} \not x\right) \\
& -i g_{s} \int_{0}^{1} d u\left[\frac{\not x}{16 \pi^{2} x^{2}} G_{\mu \nu}(u x) \sigma_{\mu \nu}-\frac{i}{4 \pi^{2} x^{2}} u x^{\mu} G_{\mu \nu}(u x) \gamma^{\nu}\right. \\
& \left.-i \frac{m_{q}}{32 \pi^{2}} G_{\mu \nu} \sigma^{\mu \nu}\left(\ln \left(\frac{-x^{2} \Lambda^{2}}{4}\right)+2 \gamma_{E}\right)\right]
\end{aligned}
$$

where $\gamma_{E} \simeq 0.577$ is the Euler constant, and $\Lambda$ is the scale parameter. In further numerical calculations, we choose it as $\Lambda=(0.5 \div 1) \mathrm{GeV}$ (see [21,22]). The heavy quark propagator in an external field has the following form:

$S_{Q}(x)=S_{Q}^{f r e e}(x)-i g_{s} \int \frac{d^{4} k}{(2 \pi)^{4}} e^{-i k x} \int_{0}^{1} d u\left[\frac{\not k+m_{Q}}{2\left(m_{Q}^{2}-k^{2}\right)^{2}} G^{\mu \nu}(u x) \sigma_{\mu \nu}+\frac{u}{m_{Q}^{2}-k^{2}} x_{\mu} G^{\mu \nu} \gamma_{\nu}\right]$

where $S_{Q}^{f r e e}(x)$ is the free heavy quark operator in x-representation, which is given by:

$$
S_{Q}^{f r e e}(x)=\frac{m_{Q}^{2}}{4 \pi^{2}} \frac{K_{1}\left(m_{Q} \sqrt{-x^{2}}\right)}{\sqrt{-x^{2}}}-i \frac{m_{Q}^{2} \not}{4 \pi^{2} x^{2}} K_{2}\left(m_{Q} \sqrt{-x^{2}}\right),
$$

where $K_{1}$ and $K_{2}$ are the modified Bessel function of the second kind.

Using the explicit expressions of the heavy and light quark propagators, as well as, definition of the DAs of the pseudoscalar mesons, the correlation function can be calculated from the QCD side. Choosing the coefficient of the structure $\not q_{\mu}$ from both sides of the correlation function and applying double Borel transformations with respect to the variables $-p^{2}$ and $-(p+q)^{2}$, in order to suppress the contributions of higher states and continuum, we get the sum rules for the strong coupling constants of pseudoscalar mesons with sextet spin-3/2 and spin-1/2 heavy baryons as:

$$
g=\frac{1}{\lambda_{1} \lambda_{2}^{(i)} m_{2}} e^{\frac{m_{1}^{2}}{M_{1}^{2}}+\frac{m_{2}^{2}}{M_{2}^{2}}+\frac{m_{P}^{2}}{M_{1}^{2}+M_{2}^{2}}} \Pi_{1}^{(i)}
$$

where $M_{1}^{2}$ and $M_{2}^{2}$ are the Borel masses in the initial and final channels, respectively. The masses of initial and final heavy baryons are very close to each other, so that we can choose $M_{1}^{2}=M_{2}^{2}=2 M^{2}$. The residues $\lambda_{1}$ of spin-3/2 and $\lambda_{2}$ of spin-1/2 are calculated in [23]. The explicit expressions for $\Pi_{1}^{(i)}$ are quite lengthy, so as an example, we present only the $\Pi_{1}^{(1)}$, which is obtained as:

$$
\begin{aligned}
& e^{m_{Q}^{2} / M^{2}-m_{\mathcal{P}}^{2} / M^{2}} \Pi_{1}^{(1)}(u, d, b)= \\
- & \frac{(1+\beta)}{8 \sqrt{6} \pi^{2}} M^{4} \mu_{\mathcal{P}}\left\{2 i_{2}(\mathcal{T}, 1)-m_{Q}^{2}\left[i_{2}(\mathcal{T}, 1)-i_{2}(\mathcal{T}, v)\right] I_{2}\right\} \\
+ & \frac{(1-\beta)}{16 \sqrt{6} \pi^{2}} M^{4} m_{Q}^{3}\left\{2 f_{\mathcal{P}} \phi_{\eta}\left(u_{0}\right) I_{2}+m_{Q}\left(\mu_{\mathcal{P}}\left[i_{3}(\mathcal{T}, 1)-2 i_{3}(\mathcal{T}, v)\right]-2 f_{\mathcal{P}} m_{Q} \phi_{\eta}\left(u_{0}\right)\right) I_{3}\right\}
\end{aligned}
$$




$$
\begin{aligned}
& +\frac{1}{96 \sqrt{6} \pi^{2}} M^{4} \mu_{\mathcal{P}}\left\{12(1+3 \beta) i_{2}(\mathcal{T}, v)+12 \beta m_{Q}^{2}\left[i_{3}(\mathcal{T}, 1)-i_{3}(\mathcal{T}, v)\right] I_{2}\right. \\
& \left.-\beta m_{Q}^{4}\left[\left(6 \phi_{\mathcal{P}}\left(u_{0}\right)+\left(1-\widetilde{\mu}_{\mathcal{P}}^{2}\right)\left[4 \phi_{\sigma}\left(u_{0}\right)-\phi_{\sigma}^{\prime}\left(u_{0}\right)\right]\right) I_{3}-m_{Q}^{2}\left(6 \phi_{\mathcal{P}}\left(u_{0}\right)-\left(1-\widetilde{\mu}_{\mathcal{P}}^{2}\right) \phi_{\sigma}^{\prime}\left(u_{0}\right)\right) I_{4}\right]\right\} \\
& -\frac{1}{16 \sqrt{6} \pi^{2}} M^{2} m_{Q} m_{\mathcal{P}}^{2} f_{\mathcal{P}}\left\{4 i_{1}\left(\mathcal{A}_{\|}, 1\right)+4 i_{1}\left(\mathcal{A}_{\perp}, 1\right)-i_{2}\left(\mathcal{A}_{\|}, 1\right)-2 i_{2}\left(\mathcal{V}_{\perp}, 1\right)\right. \\
& \left.-\beta\left[4 i_{1}\left(\mathcal{V}_{\|}, 1\right)+4 i_{1}\left(\mathcal{V}_{\perp}, 1\right)+i_{2}\left(\mathcal{A}_{\|}, 1\right)-4 i_{2}\left(\mathcal{A}_{\perp}, 1\right)-2 i_{2}\left(\mathcal{V}_{\|}, 1\right)+2 i_{2}\left(\mathcal{V}_{\perp}, 1\right)\right]\right\} I_{1} \\
& +\frac{1}{32 \sqrt{6} \pi^{2}} M^{2} m_{Q}^{2} m_{\mathcal{P}}^{2}\left\{-4 \beta m_{Q} f_{\mathcal{P}} i_{2}\left(\mathcal{A}_{\|}, 1\right)+8 \beta m_{Q} f_{\mathcal{P}} i_{2}\left(\mathcal{A}_{\perp}, 1\right)+9 \beta \mu_{\mathcal{P}} i_{2}(\mathcal{T}, 1)\right. \\
& -2(1-3 \beta) m_{Q} f_{\mathcal{P}} i_{2}\left(\mathcal{V}_{\|}, 1\right)-4(1+\beta) m_{Q} f_{\mathcal{P}} i_{2}\left(\mathcal{V}_{\perp}, 1\right)-4(1-\beta) m_{Q} f_{\mathcal{P}} i_{2}\left(\mathcal{A}_{\|}, v\right) \\
& \left.+\mu_{\mathcal{P}}(1-5 \beta) i_{2}(\mathcal{T}, v)-m_{Q} f_{\mathcal{P}}\left[(1-\beta) \mathbb{A}\left(u_{0}\right)+2(1+\beta) \widetilde{i}_{4}(\mathbb{B})\right]\right\} I_{2} \\
& +\frac{1}{12 \sqrt{6} \pi^{2}} M^{2} f_{\mathcal{P}}\left\{-3(1+\beta) m_{Q} m_{\mathcal{P}}^{2}\left[i_{1}\left(\mathcal{A}_{\|}, 1\right)+i_{1}\left(\mathcal{A}_{\perp}, 1\right)+i_{1}\left(\mathcal{V}_{\|}, 1\right)+i_{1}\left(\mathcal{V}_{\perp}, 1\right)\right.\right. \\
& \left.\left.-2\left(i_{1}\left(\mathcal{A}_{\|}, v\right)+i_{1}\left(\mathcal{A}_{\perp}, v\right)\right)\right]+2(2+\beta) \pi^{2}\langle\bar{d} d\rangle \phi_{\eta}\left(u_{0}\right)\right\} \\
& +\frac{1}{48 \sqrt{6} \pi^{2}} M^{2} m_{Q}^{6} m_{\mathcal{P}}^{2} \mu_{\mathcal{P}}\left(1-\widetilde{\mu}_{\mathcal{P}}^{2}\right) \beta \phi_{\sigma}\left(u_{0}\right) I_{4} \\
& +\frac{1}{96 \sqrt{6} \pi^{2}} M^{2} m_{Q}^{4} m_{\mathcal{P}}^{2}\left\{15(1-\beta) \mu_{\mathcal{P}} i_{2}(\mathcal{T}, 1)-6(5-2 \beta) \mu_{\mathcal{P}} i_{2}(\mathcal{T}, v)\right. \\
& \left.+6(1+\beta) m_{Q} f_{\mathcal{P}} \widetilde{i}_{4}(\mathbb{B})-2 \beta \mu_{\mathcal{P}}\left(1-\widetilde{\mu}_{\mathcal{P}}^{2}\right) \phi_{\sigma}\left(u_{0}\right)\right\} I_{3} \\
& +\frac{1}{96 \sqrt{6} M^{6}} m_{Q}^{4} m_{\mathcal{P}}^{2} m_{0}^{2} f_{\mathcal{P}}\langle\bar{d} d\rangle\left\{(2+\beta) \mathbb{A}\left(u_{0}\right)+8\left[i_{1}\left(\mathcal{A}_{\|}, 1\right)+i_{1}\left(\mathcal{A}_{\perp}, 1\right)\right.\right. \\
& \left.\left.+\beta i_{1}\left(\mathcal{V}_{\|}, 1\right)+\beta i_{1}\left(\mathcal{V}_{\perp}, 1\right)-2 i_{1}\left(\mathcal{A}_{\|}, v\right)-2 i_{1}\left(\mathcal{A}_{\perp}, v\right)\right]\right\} \\
& -\frac{1}{192 \sqrt{6} M^{4}} m_{Q}^{2} m_{\mathcal{P}}^{2} m_{0}^{2} f_{\mathcal{P}}\langle\bar{d} d\rangle\left\{\mathbb{A}\left(u_{0}\right)+32\left[i_{1}\left(\mathcal{A}_{\|}, 1\right)+i_{1}\left(\mathcal{A}_{\perp}, 1\right)+\beta i_{1}\left(\mathcal{V}_{\|}, 1\right)\right.\right. \\
& \left.+\beta i_{1}\left(\mathcal{V}_{\perp}, 1\right)-2 i_{1}\left(\mathcal{A}_{\|}, v\right)-2 i_{1}\left(\mathcal{A}_{\perp}, v\right)\right]+4(1+2 \beta)\left[2 i_{2}\left(\mathcal{A}_{\perp}, 1\right)+i_{2}\left(\mathcal{V}_{\|}, 1\right)\right] \\
& \left.+4(2+\beta)\left[i_{2}\left(\mathcal{A}_{\|}, 1\right)+2 i_{2}\left(\mathcal{V}_{\perp}, 1\right)-2 i_{2}\left(\mathcal{A}_{\|}, v\right)-4 i_{2}\left(\mathcal{V}_{\perp}, v\right)\right]-4 \widetilde{i}_{4}(\mathbb{B})\right\} \\
& -\frac{(1-\beta)}{72 \sqrt{6} M^{4}} m_{Q}^{3} m_{0}^{2} \mu_{\mathcal{P}}\langle\bar{d} d\rangle\left[3 i_{2}(\mathcal{T}, 1)-\left(1-\widetilde{\mu}_{\mathcal{P}}^{2}\right) \phi_{\sigma}\left(u_{0}\right)\right] \\
& -\frac{1}{3 \sqrt{6} M^{2}} m_{Q}^{2} m_{\mathcal{P}}^{2} f_{\mathcal{P}}\langle\bar{d} d\rangle\left\{i_{1}\left(\mathcal{A}_{\|}, 1\right)+i_{1}\left(\mathcal{A}_{\perp}, 1\right)+\beta\left[i_{1}\left(\mathcal{V}_{\|}, 1\right)+i_{1}\left(\mathcal{V}_{\perp}, 1\right)\right]\right. \\
& \left.-2\left[i_{1}\left(\mathcal{A}_{\|}, v\right)+i_{1}\left(\mathcal{A}_{\perp}, v\right)\right]\right\} \\
& +\frac{1}{288 \sqrt{6} M^{2}}\left\{12(1-\beta) m_{Q} \mu_{\mathcal{P}} m_{0}^{2}\langle\bar{d} d\rangle i_{2}(\mathcal{T}, 1)+(2-\beta) f_{\mathcal{P}} m_{\mathcal{P}}^{2} m_{0}^{2}\langle\bar{d} d\rangle \widetilde{i}_{4}(\mathbb{B})\right. \\
& \left.-12(2+\beta) m_{Q}^{2} f_{\mathcal{P}}\langle\bar{d} d\rangle\left[m_{\mathcal{P}}^{2} \mathbb{A}\left(u_{0}\right)+m_{0}^{2} \phi_{\eta}\left(u_{0}\right)\right]\right\} \\
& +\frac{\beta}{16 \sqrt{6} \pi^{2}} m_{Q} m_{\mathcal{P}}^{4} f_{\mathcal{P}}\left\{\left[i_{1}\left(\mathcal{A}_{\|}, 1\right)+i_{1}\left(\mathcal{A}_{\perp}, 1\right)-i_{1}\left(\mathcal{V}_{\|}, 1\right)-i_{1}\left(\mathcal{V}_{\perp}, 1\right)\right]\left(m_{Q}^{2} I_{2}-I_{1}\right)\right\}
\end{aligned}
$$




$$
\begin{aligned}
& +\frac{1}{12 \sqrt{6}}\langle\bar{d} d\rangle\left\{2(1-\beta) m_{Q} \mu_{\mathcal{P}} i_{2}(\mathcal{T}, 1)+(1+2 \beta) m_{\mathcal{P}}^{2} f_{\mathcal{P}}\left[2 i_{2}\left(\mathcal{A}_{\perp}, 1\right)+i_{2}\left(\mathcal{V}_{\|}, 1\right)\right]\right. \\
& \left.+(2+\beta) m_{\mathcal{P}}^{2} f_{\mathcal{P}}\left[i_{2}\left(\mathcal{A}_{\|}, 1\right)+2 i_{2}\left(\mathcal{V}_{\perp}, 1\right)-2 i_{2}\left(\mathcal{A}_{\|}, v\right)-4 i_{2}\left(\mathcal{V}_{\perp}, v\right)\right]\right\} \\
& -\frac{1}{144 \sqrt{6}}\langle\bar{d} d\rangle\left\{6 m_{\mathcal{P}}^{2} f_{\mathcal{P}}\left[(2+\beta) \mathbb{A}\left(u_{0}\right)+2 \widetilde{i}_{4}(\mathbb{B})\right]+3(3+2 \beta) m_{0}^{2} f_{\mathcal{P}} \phi_{\eta}\left(u_{0}\right)\right. \\
& \left.+8(1-\beta) m_{Q} \mu_{\mathcal{P}}\left(1-\widetilde{\mu}_{\mathcal{P}}^{2}\right) \phi_{\sigma}\left(u_{0}\right)\right\}
\end{aligned}
$$

where,

$$
\begin{aligned}
i_{1}(\phi, f(v)) & =\int \mathcal{D} \alpha_{i} \int_{0}^{1} d v \phi\left(\alpha_{\bar{q}}, \alpha_{q}, \alpha_{g}\right) f(v) \theta\left(k-u_{0}\right), \\
i_{2}(\phi, f(v)) & =\int \mathcal{D} \alpha_{i} \int_{0}^{1} d v \phi\left(\alpha_{\bar{q}}, \alpha_{q}, \alpha_{g}\right) f(v) \delta\left(k-u_{0}\right), \\
i_{3}(\phi, f(v)) & =\int \mathcal{D} \alpha_{i} \int_{0}^{1} d v \phi\left(\alpha_{\bar{q}}, \alpha_{q}, \alpha_{g}\right) f(v) \delta^{\prime}\left(k-u_{0}\right), \\
\widetilde{i}_{4}(f(u)) & =\int_{u_{0}}^{1} d u f(u), \\
I_{n} & =\int_{m_{Q}^{2}}^{\infty} d s \frac{e^{m_{Q}^{2} / M^{2}-s / M^{2}}}{s^{n}},
\end{aligned}
$$

and

$$
\begin{aligned}
k & =\alpha_{q}+\alpha_{g} \bar{v}, \quad u_{0}=\frac{M_{1}^{2}}{M_{1}^{2}+M_{2}^{2}}, \quad M^{2}=\frac{M_{1}^{2} M_{2}^{2}}{M_{1}^{2}+M_{2}^{2}}, \\
\mu_{\mathcal{P}} & =f_{\mathcal{P}} \frac{m_{\mathcal{P}}^{2}}{m_{q_{1}}+m_{q_{2}}}, \quad \widetilde{\mu}_{\mathcal{P}}=\frac{m_{q_{1}}+m_{q_{2}}}{m_{\mathcal{P}}},
\end{aligned}
$$

The $D \alpha_{i}=d \alpha_{\bar{q}} d \alpha_{q} d \alpha_{g} \delta\left(1-\alpha_{\bar{q}}-\alpha_{q}-\alpha_{g}\right), q_{1}$ and $q_{2}$ are the light quarks, $Q$ is the heavy quark, the subscript $\mathcal{P}$ stands for pseudoscalar meson and the functions $\mathcal{A}_{\|}, \mathcal{A}_{\perp}, \mathcal{T}, \mathcal{V}_{\|}$, $\mathcal{V}_{\perp}, \phi_{\sigma}, \phi_{\sigma}^{\prime}, \phi_{\eta}, \phi_{\mathcal{P}}, \mathbb{A}$ and $\mathbb{B}$ are the DAs with definite twists for the pseudoscalar mesons. To shorten the above expression, we have ignored the light quarks masses as well as terms containing gluon condensates, but we take into account their contribution when doing numerical analysis. The continuum subtraction is performed using results of the work [15].

\section{Numerical analysis}

This section is devoted to the numerical analysis of the strong coupling constants of mesons with spin $-3 / 2$ and spin $-1 / 2$ heavy baryons. The main input parameters for performing numerical analysis are the DAs of the light pseudoscalar mesons, whose expressions are presented in [19]. The other input parameters appearing in the sum rules are, $\langle\bar{q} q\rangle=$ $-(0.24 \pm 0.001)^{3} \mathrm{GeV}^{3}, m_{0}^{2}=(0.8 \pm 0.2) \mathrm{GeV}^{2}[13], f_{\pi}=0.131 \mathrm{GeV}, f_{K}=0.16 \mathrm{GeV}$ and $f_{\eta}=0.13 \mathrm{GeV}$. 


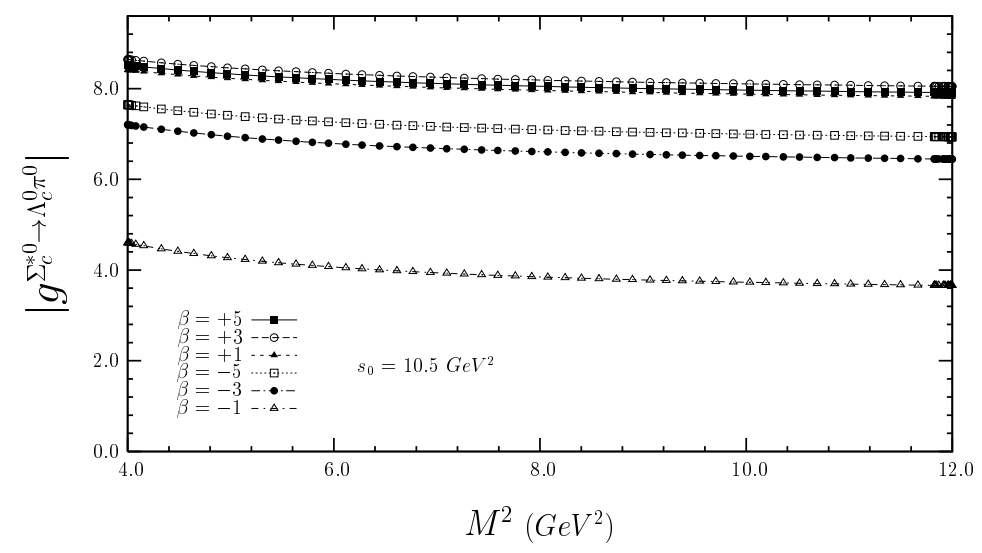

Figure 1: The dependence of the strong coupling constant for the $\Sigma_{c}^{* 0} \rightarrow \Lambda_{c}^{0} \pi^{0}$ transition on $M^{2}$ at several fixed values of $\beta$, and at $s_{0}=10.5 \mathrm{GeV}^{2}$.

In the sum rules for the strong coupling constants of light pseudoscalar mesons with heavy baryons, there are three auxiliary parameters, namely Borel mass $M^{2}$, continuum threshold $s_{0}$ and the arbitrary parameter $\beta$ in the expressions of the interpolating currents of spin $-1 / 2$ baryons. It is clear that, any physical quantity, like the aforementioned strong coupling constants, should be independent of these auxiliary parameters. Therefore, we try to find so called "working regions" of these parameters, where $g_{B^{*} B P}$ is practically independent of them. The upper limit of $M^{2}$ can be obtained by demanding that the higher states and continuum contributions contribute less than, say, $50 \%$ of the total dispersion integral. The lower bound of $M^{2}$ can be determined by requiring that the highest power in $1 / M^{2}$ should be less than $(20-25) \%$ of the highest power $M^{2}$. These two conditions allow us to fix the following working regions: $15 \mathrm{GeV}^{2} \leq \mathrm{M}^{2} \leq 30 \mathrm{GeV}^{2}$ for the bottom baryons, and $4 \mathrm{GeV}^{2} \leq M^{2} \leq 12 \mathrm{GeV}^{2}$ for the charmed baryons. As far as continuum threshold is concerned, we choose it in the interval between $s_{0}=\left(m_{B}+0.5\right)^{2} \mathrm{GeV}^{2}$ and $s_{0}=\left(m_{B}+0.7\right)^{2} \mathrm{GeV}^{2}$.

As an example, in Figs. 1 and 2, we present the dependence of the coupling constants for the $\Sigma_{c}^{* 0} \rightarrow \Lambda_{c}^{0} \pi^{0}$ and $\Xi_{c}^{*+} \rightarrow \Xi_{c}^{+} \pi^{0}$ transitions on $M^{2}$, at different fixed values of $\beta$ and at $s_{0}=10.5 \mathrm{GeV}^{2}$. From these figures, we see that the coupling constants for these transitions exhibit good stability when $M^{2}$ is varied in the above mentioned "working region". Depicted in Figs. 2 and 4 are the dependences of the same coupling constants on $\cos \theta$ at several fixed values of $s_{0}$ and at $M^{2}=8 \mathrm{GeV}^{2}$, where $\beta=\tan \theta$. From these figures, we observe that when $\cos \theta$ is varied in the domain $-0.3 \leq \cos \theta \leq 0.5$, the coupling constants show rather stable behavior and they also have very weak dependence on $s_{0}$. Similar analysis for the strong coupling constants of all $\mathrm{S}^{*} \mathrm{SP}$ and $\mathrm{S}^{*} \mathrm{AP}$ is performed and the results are presented in Tables 3 and 4, respectively. For completeness, in these Tables we also present the predictions of the Ioffe current $(\beta=-1)$ for these coupling constants. Here, we would like to remind that, our obtained domain for $\cos \theta$ lies inside the more wide interval obtained from analysis of mass sum rules for heavy non strange baryons in [18, 24].

Note that only few of the presented coupling constants can be measured directly from the analysis of the decays, and the remaining coupling constants can only be measured, 
indirectly. At present, the decay widths of the $\Sigma_{c}^{*++} \rightarrow \Lambda_{c}^{+} \pi^{+}$and $\Sigma_{c}^{* 0} \rightarrow \Lambda_{c}^{+} \pi^{-}$are measured, experimentally and also the upper bounds for the $\Sigma_{c}^{*+} \rightarrow \Lambda_{c}^{+} \pi^{0}, \Xi_{c}^{*+} \rightarrow \Xi_{c}^{0} \pi^{+}$, $\Xi_{c}^{*+} \rightarrow \Xi_{c}^{+} \pi^{0}, \Xi_{c}^{* 0} \rightarrow \Xi_{c}^{+} \pi^{-}$and $\Xi_{c}^{* 0} \rightarrow \Xi_{c}^{0} \pi^{0}$ are announced (see [4] and [6]). Using the matrix element for the $3 / 2 \rightarrow 1 / 2 \pi$ transition, i.e.,

$$
\mathcal{M}=g_{B^{*} B \pi} \bar{u}(p) u^{\alpha}(p+q) q_{\alpha},
$$

one can easily obtain the following relation for the corresponding decay width:

$$
\Gamma=\frac{g^{2}}{24 \pi m_{1}^{2}}|\vec{q}|^{3}\left[\left(m_{1}+m_{2}\right)^{2}-m_{\pi}^{2}\right],
$$

where $|\vec{q}|$ is the momentum of the $\pi$ meson. Using the values of the coupling constants from Tables (3) and (4), and also Eq. (30), we can easily predict the values of the corresponding decay widths. Our predictions on these decays, the experimental results, as well as predictions of other approaches on these coupling constants are presented in Table 5. From this Table, we see that our predictions on decay widths for the above-mentioned kinematically allowed transitions are all in good agreement with the existing experimental results and the prediction of other approaches.

In summary, we calculated the strong coupling constants of spin- $3 / 2$ to spin $-1 / 2$ transitions with the participation of pseudoscalar mesons within LCSR. Our analysis shows that all $\mathrm{S}^{*} \mathrm{SP}$ and $\mathrm{S}^{*} \mathrm{AP}$ couplings are described by only one invariant function in each class of transitions. Moreover, we estimated the widths of the kinematically allowed transitions, which match quite good with the existing experimental data, as well as predictions of other approaches.

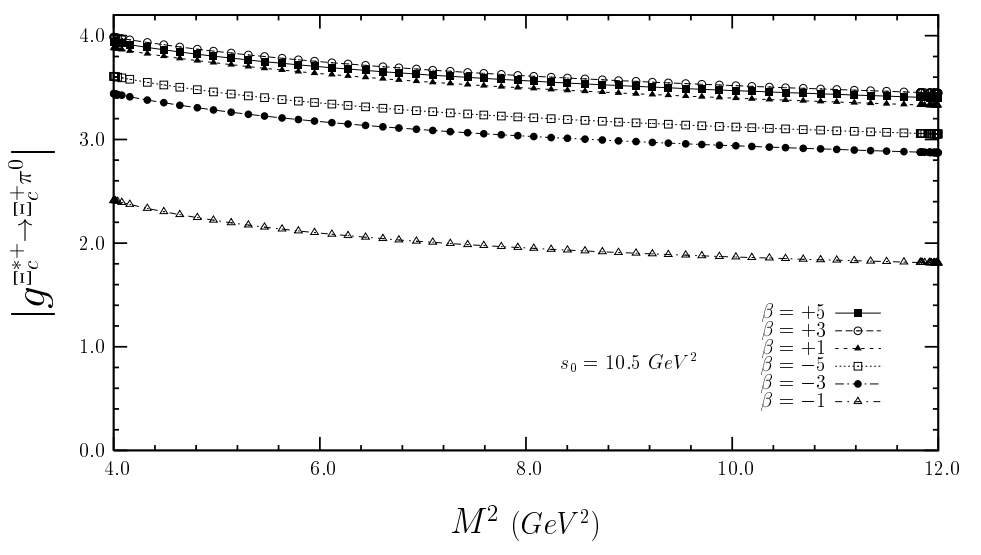

Figure 2: The dependence of the strong coupling constant for the $\Xi_{c}^{*+} \rightarrow \Xi_{c}^{+} \pi^{0}$ transition on $M^{2}$ at several fixed values of $\beta$, and at $s_{0}=10.5 \mathrm{GeV}^{2}$. 


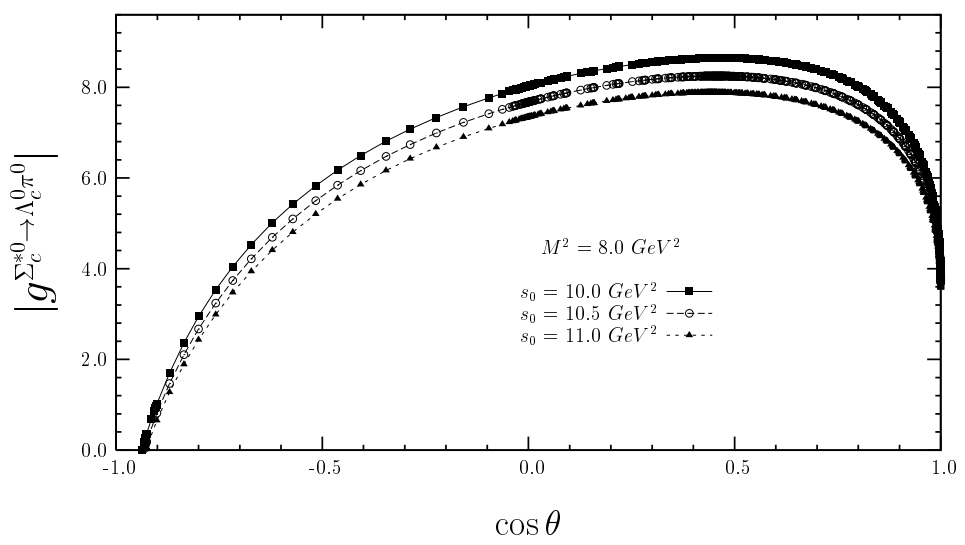

Figure 3: The dependence of the strong coupling constant for the $\Sigma_{c}^{* 0} \rightarrow \Lambda_{c}^{0} \pi^{0}$ transition on $\cos \theta$ at several fixed values of $s_{0}$, and at $M^{2}=8.0 \mathrm{GeV}^{2}$.

\section{Appendix A :}

In this appendix we present the expressions of the correlation functions in terms of invariant function $\Pi_{1}^{(1)}$ and $\Pi_{1}^{(2)}$ involving $\pi, K$ and $\eta_{1}$ mesons.

- Correlation functions describing pseudoscalar mesons with sextet-sextet baryons.

$$
\begin{aligned}
\Pi^{\Sigma_{b}^{*+} \rightarrow \Sigma_{b}^{+} \pi^{0}} & =\sqrt{2} \Pi_{1}^{(1)}(u, u, b) \\
\Pi^{\Sigma_{b}^{*-} \rightarrow \Sigma_{b}^{-} \pi^{0}} & =-\sqrt{2} \Pi_{1}^{(1)}(d, d, b) \\
\Pi_{b}^{\Sigma_{b}^{*+} \rightarrow \Sigma_{b}^{0} \pi^{+}} & =\sqrt{2} \Pi_{1}^{(1)}(d, u, b) \\
\Pi^{\Sigma_{b}^{* 0} \rightarrow \Sigma_{b}^{-} \pi^{+}} & =\sqrt{2} \Pi_{1}^{(1)}(u, d, b)
\end{aligned}
$$

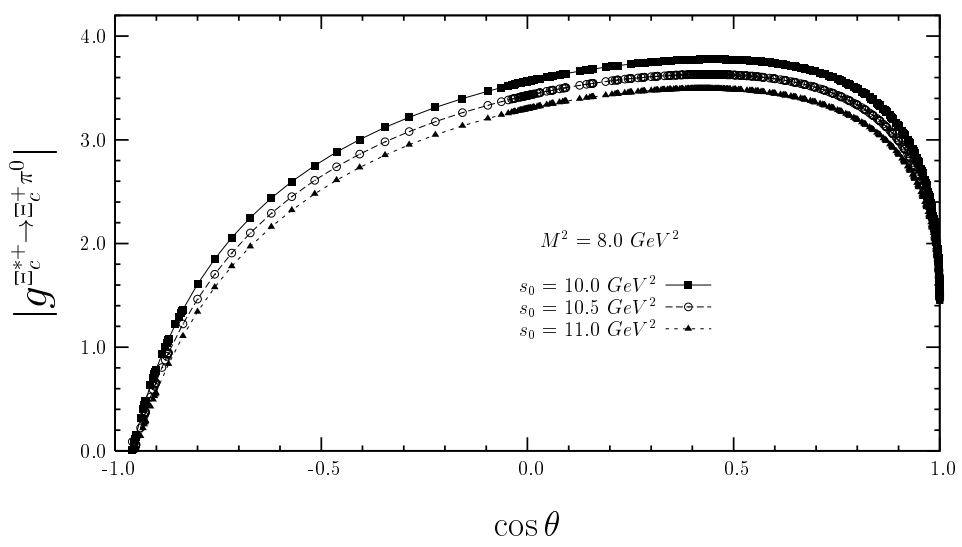

Figure 4: The dependence of the strong coupling constant for the $\Xi_{c}^{*+} \rightarrow \Xi_{c}^{+} \pi^{0}$ transition on $\cos \theta$ at several fixed values of $s_{0}$, and at $M^{2}=8.0 \mathrm{GeV}^{2}$. 


\begin{tabular}{|c|c|c|c|c|c|}
\hline \multirow{2}{*}{$g$ channel } & \multicolumn{2}{|c|}{ Bottom Baryons } & \multirow{2}{*}{$g$ channel } & \multicolumn{2}{|c|}{ Charmed Baryons } \\
\hline & General current & Ioffe current & & General current & Ioffe current \\
\hline$g^{\Xi_{b}^{* 0} \rightarrow \Xi_{b}^{\prime} \pi^{0}}$ & $2.0 \pm 0.4$ & $1.6 \pm 0.4$ & $g^{\Xi_{c}^{*+} \rightarrow \Xi_{c}^{+} \pi^{0}}$ & $2.1 \pm 0.3$ & $2.3 \pm 0.4$ \\
\hline$g^{\Sigma_{b}^{* 0} \rightarrow \Sigma_{b}^{-} \pi^{+}}$ & $3.7 \pm 0.6$ & $3.5 \pm 0.5$ & $g^{\Sigma_{c}^{*+} \rightarrow \Sigma_{c}^{0} \pi^{+}}$ & $4.2 \pm 0.5$ & $4.3 \pm 0.4$ \\
\hline$g^{\Xi_{b}^{* 0} \rightarrow \Sigma_{b}^{+} K^{-}}$ & $4.8 \pm 1.0$ & $2.6 \pm 0.5$ & $g^{\Xi_{c}^{*+} \rightarrow \Sigma_{c}^{++}} K^{-}$ & $4.5 \pm 0.5$ & $3.9 \pm 0.4$ \\
\hline$g^{\Omega_{b}^{*-}} \rightarrow \Xi_{b}^{\prime 0} K^{-}$ & $5.0 \pm 1.4$ & $2.5 \pm 0.4$ & $g^{\Omega_{c}^{* 0} \rightarrow \Xi_{c}^{\prime}} K^{-}$ & $4.3 \pm 0.5$ & $4.5 \pm 0.4$ \\
\hline$g^{\Sigma_{b}^{*+}} \rightarrow \Sigma_{b}^{+} \eta_{1}$ & $2.9 \pm 0.7$ & $2.0 \pm 0.4$ & $g^{\Sigma_{c}^{*++} \rightarrow \Sigma_{c}^{++}} \eta_{1}$ & $2.9 \pm 0.4$ & $2.7 \pm 0.4$ \\
\hline$g^{\Xi_{b}^{* 0} \rightarrow \Xi_{b}^{\prime 0} \eta_{1}}$ & $1.3 \pm 0.3$ & $0.9 \pm 0.3$ & $g^{\Xi_{c}^{*+} \rightarrow \Xi_{c}^{\prime+} \eta_{1}}$ & $1.5 \pm 0.3$ & $1.0 \pm 0.2$ \\
\hline$g^{\Omega_{b}^{*-} \rightarrow \Omega_{b}^{-} \eta_{1}}$ & $5.8 \pm 1.6$ & $3.6 \pm 0.6$ & $g^{\Omega_{c}^{* 0} \rightarrow \Omega_{c}^{0} \eta_{1}}$ & $5.9 \pm 0.8$ & $5.8 \pm 0.4$ \\
\hline
\end{tabular}

Table 3: Values of the strong coupling constants $g$ in $\mathrm{GeV}^{-1}$ for the transitions among the sextet spin- $3 / 2$ and sextet spin-1/2 heavy baryons with pseudoscalar mesons.

\begin{tabular}{|c|c|c|c|c|c|}
\hline \multirow{2}{*}{$g$ channel } & \multicolumn{2}{|c|}{ Bottom Baryons } & \multirow{2}{*}{$g$ channel } & \multicolumn{2}{|c|}{ Charmed Baryons } \\
\hline & General current & Ioffe current & & General current & Ioffe current \\
\hline$g^{\Xi_{b}^{* 0} \rightarrow \Xi_{b}^{0} \pi^{0}}$ & $3.0 \pm 0.6$ & $1.4 \pm 0.3$ & $g^{\Xi_{c}^{*+} \rightarrow \Xi_{c}^{+} \pi^{0}}$ & $3.5 \pm 0.5$ & $2.0 \pm 0.3$ \\
\hline$g^{\Sigma_{b}^{*-} \rightarrow \Lambda_{b}^{0} \pi^{-}}$ & $6.0 \pm 1.1$ & $2.5 \pm 0.5$ & $g^{\Sigma_{c}^{* 0} \rightarrow \Lambda_{c}^{+} \pi^{-}}$ & $7.8 \pm 1.0$ & $3.9 \pm 0.6$ \\
\hline$g^{\Sigma_{b}^{* 0} \rightarrow \Xi_{b}^{0} \bar{K}^{0}}$ & $3.7 \pm 0.5$ & $2.0 \pm 0.5$ & $g^{\Sigma_{c}^{*+} \rightarrow \Xi_{c}^{+} \bar{K}^{0}}$ & $5.0 \pm 1.0$ & $3.1 \pm 0.4$ \\
\hline$g^{\Omega_{b}^{*-} \rightarrow \Xi_{b}^{-} \bar{K}^{0}}$ & $5.0 \pm 0.8$ & $2.6 \pm 0.4$ & $g^{\Omega_{c}^{* 0} \rightarrow \Xi_{c}^{0} \bar{K}^{0}}$ & $6.2 \pm 1.5$ & $4.1 \pm 0.5$ \\
\hline$g^{\Xi_{b}^{* 0} \rightarrow \Xi_{b}^{-} K^{+}}$ & $3.6 \pm 0.5$ & $1.9 \pm 0.6$ & $g^{\Xi_{c}^{*+}} \rightarrow \Xi_{c}^{0} K^{+}$ & $4.4 \pm 0.8$ & $3.0 \pm 0.4$ \\
\hline$g^{\Xi_{b}^{* 0} \rightarrow \Xi_{b}^{0} \eta_{1}}$ & $5.4 \pm 1.0$ & $2.5 \pm 0.4$ & $g^{\Xi_{c}^{*+} \rightarrow \Xi_{c}^{+} \eta_{1}}$ & $6.9 \pm 1.5$ & $4.0 \pm 0.5$ \\
\hline
\end{tabular}

Table 4: Values of the strong coupling constants $g$ in $\mathrm{GeV}^{-1}$ for the transitions among the sextet spin-3/2 and anti-triplet spin-1/2 heavy baryons with pseudoscalar mesons.

$$
\begin{aligned}
\Pi^{\Xi_{b}^{* 0} \rightarrow \Xi_{b}^{\prime}-\pi^{+}} & =\Pi_{1}^{(1)}(d, s, b), \\
\Pi_{b}^{\Sigma_{b}^{* 0} \rightarrow \Sigma_{b}^{+} \pi^{-}} & =\sqrt{2} \Pi_{1}^{(1)}(d, u, b), \\
\Pi^{\Sigma_{b}^{*-} \rightarrow \Sigma_{b}^{0} \pi^{-}} & =\sqrt{2} \Pi_{1}^{(1)}(u, d, b), \\
\Pi^{\Xi_{b}^{*-} \rightarrow \Xi_{b}^{\prime 0} \pi^{-}} & =\Pi_{1}^{(1)}(u, s, b), \\
\Pi^{\Xi_{b}^{* 0} \rightarrow \Sigma_{b}^{+} K^{-}} & =\sqrt{2} \Pi_{1}^{(1)}(u, u, b), \\
\Pi^{\Xi_{b}^{*-} \rightarrow \Sigma_{b}^{0} K^{-}} & =\sqrt{2} \Pi_{1}^{(1)}(u, d, b), \\
\Pi^{\Omega_{b}^{*-} \rightarrow \Xi_{b}^{\prime} K^{-}} & =\sqrt{2} \Pi_{1}^{(1)}(s, s, b), \\
\Pi^{\Sigma_{b}^{*+} \rightarrow \Xi_{b}^{\prime 0} K^{+}} & =\sqrt{2} \Pi_{1}^{(1)}(u, u, b), \\
\Pi^{\Sigma_{b}^{* 0} \rightarrow \Xi_{b}^{\prime}-} K^{+} & =\Pi_{1}^{(1)}(u, d, b),
\end{aligned}
$$




\begin{tabular}{|l|c|c|c|c|c|c|}
\hline \hline & Our work & CQM [25] & LFQM [26] & RQM [27] & NRQM [28] & Experiment[29] \\
\hline$\Gamma\left(\Sigma_{c}^{*++} \rightarrow \Lambda_{c}^{+} \pi^{+}\right)$ & $14.6 \pm 3.8$ & 20 & 12.84 & $21.90 \pm 0.87$ & $17.52 \pm 0.74$ & $14.9 \pm 1.9$ \\
$\Gamma\left(\Sigma_{c}^{*+} \rightarrow \Lambda_{c}^{+} \pi^{0}\right)$ & $14.6 \pm 3.8$ & 20 & - & - & - & $<17$ \\
$\Gamma\left(\Sigma_{c}^{* 0} \rightarrow \Lambda_{c}^{0} \pi^{0}\right)$ & $14.6 \pm 3.8$ & 20 & 12.40 & $21.20 \pm 0.81$ & $16.90 \pm 0.71$ & $16.1 \pm 2.1$ \\
$\Gamma\left(\Xi_{c}^{*+} \rightarrow \Xi_{c}^{0} \pi^{+}\right)$ & $2.8 \pm 0.9$ & - & 1.12 & $1.78 \pm 0.33$ & - & $<3.1$ \\
$\Gamma\left(\Xi_{c}^{*+} \rightarrow \Xi_{c}^{+} \pi^{0}\right)$ & $1.4 \pm 0.4$ & - & 0.69 & $1.26 \pm 0.17$ & - & $<3.1$ \\
$\Gamma\left(\Xi_{c}^{* 0} \rightarrow \Xi_{c}^{+} \pi^{-}\right)$ & $2.8 \pm 0.9$ & - & 1.16 & $2.11 \pm 0.29$ & - & $<5.5$ \\
$\Gamma\left(\Xi_{c}^{* 0} \rightarrow \Xi_{c}^{0} \pi^{0}\right)$ & $1.4 \pm 0.4$ & - & 0.72 & $1.01 \pm 0.15$ & - & $<5.5$ \\
\hline \hline
\end{tabular}

Table 5: Strong one-pion decay rates. Here the short keys stand for: (CQM) Constituent Quark Model, (LFQM) Light-Front Quark Model, (RQM) Relativistic Quark Model, (NRQM) Non-Relativistic Quark Model. The results are presented in units of $\mathrm{MeV}$.

$$
\begin{aligned}
& \Pi^{\Xi_{b}^{* 0} \rightarrow \Omega_{b}^{-} K^{+}}=\sqrt{2} \Pi_{1}^{(1)}(s, s, b), \\
& \Pi^{\Xi_{b}^{* 0} \rightarrow \Sigma_{b}^{0} \bar{K}^{0}}=\Pi_{1}^{(1)}(d, u, b), \\
& \Pi^{\Xi_{b}^{*-} \rightarrow \Sigma_{b}^{-} \bar{K}^{0}}=\sqrt{2} \Pi_{1}^{(1)}(d, d, b), \\
& \Pi^{\Omega_{b}^{*-} \rightarrow \Xi_{b}^{\prime}-\bar{K}^{0}}=\sqrt{2} \Pi_{1}^{(1)}(s, s, b), \\
& \Pi^{\Sigma_{b}^{* 0} \rightarrow \Xi_{b}^{\prime 0} K^{0}}=\Pi_{1}^{(1)}(d, u, b), \\
& \Pi^{\Sigma_{b}^{*-} \rightarrow \Xi_{b}^{\prime}-K^{0}}=\sqrt{2} \Pi_{1}^{(1)}(d, d, b) \text {, } \\
& \Pi^{\Xi_{b}^{*-} \rightarrow \Omega_{b}^{-} K^{0}}=\sqrt{2} \Pi_{1}^{(1)}(s, s, b), \\
& \Pi^{\Sigma_{b}^{*+} \rightarrow \Sigma_{b}^{+} \eta_{1}}=\frac{2}{\sqrt{6}} \Pi_{1}^{(1)}(u, u, b), \\
& \Pi^{\Sigma_{b}^{*-} \rightarrow \Sigma_{b}^{-}} \eta_{1}=\frac{2}{\sqrt{6}} \Pi_{1}^{(1)}(d, d, b), \\
& \Pi^{\Xi_{b}^{*-} \rightarrow \Xi_{b}^{\prime}-} \eta_{1}=\frac{1}{\sqrt{6}}\left[\Pi_{1}^{(1)}(d, s, b)-2 \Pi_{1}^{(1)}(s, d, b)\right], \\
& \Pi^{\Omega_{b}^{*-} \rightarrow \Omega_{b}^{-} \eta_{1}}=-\frac{4}{\sqrt{6}} \Pi_{1}^{(1)}(s, s, b) \text {. }
\end{aligned}
$$

- Correlation functions responsible for the transitions of the sextet-anti-triplet baryons.

$$
\begin{aligned}
\Pi^{\Xi_{b}^{*-} \rightarrow \Xi_{b}^{0} \pi^{-}} & =\Pi_{1}^{(2)}(d, s, b), \\
\Pi^{\Xi_{b}^{* 0} \rightarrow \Xi_{b}^{-} \pi^{+}} & =\Pi_{1}^{(2)}(u, s, b), \\
\Pi^{\Sigma_{b}^{* 0} \rightarrow \Xi_{b}^{0} \bar{K}^{0}} & =-\Pi_{1}^{(2)}(d, u, b), \\
\Pi^{\Sigma_{b}^{*-} \rightarrow \Xi_{b}^{-}} \bar{K}^{0} & =-\Pi_{1}^{(2)}(d, d, b), \\
\Pi^{\Omega_{b}^{*-} \rightarrow \Xi_{b}^{-}} \bar{K}^{0} & =\sqrt{2} \Pi_{1}^{(2)}(s, s, b),
\end{aligned}
$$




$$
\begin{aligned}
& \Pi^{\Xi_{b}^{* 0} \rightarrow \Lambda_{b}^{0} \bar{K}^{0}}=-\Pi_{1}^{(2)}(d, u, b), \\
& \Pi^{\Sigma_{b}^{* 0} \rightarrow \Xi_{b}^{0} K^{0}}=-\Pi_{1}^{(2)}(d, u, b), \\
& \Pi^{\Sigma_{b}^{*-} \rightarrow \Xi_{b}^{-} K^{0}}=-\sqrt{2} \Pi_{1}^{(2)}(d, d, b), \\
& \Pi^{\Omega_{b}^{*-} \rightarrow \Xi_{b}^{-} K^{0}}=\sqrt{2} \Pi_{1}^{(2)}(s, s, b), \\
& \Pi^{\Xi_{b}^{* 0} \rightarrow \Lambda_{b}^{0} K^{0}}=-\Pi_{1}^{(2)}(d, u, b), \\
& \Pi^{\Sigma_{b}^{*+} \rightarrow \Lambda_{b}^{0} K^{+}}=-\sqrt{2} \Pi_{1}^{(2)}(u, u, b), \\
& \Pi^{\Sigma_{b}^{* 0} \rightarrow \Xi_{b}^{-} K^{+}}=-\Pi_{1}^{(2)}(u, d, b), \\
& \Pi^{\Xi_{b}^{* 0} \rightarrow \Xi_{b}^{-} K^{+}}=\Pi_{1}^{(2)}(d, s, b), \\
& \Pi^{\Sigma_{b}^{*-} \rightarrow \Lambda_{b}^{0} K^{-}}=\sqrt{2} \Pi_{1}^{(2)}(d, d, b), \\
& \Pi^{\Omega_{b}^{*-} \rightarrow \Xi_{b}^{0} K^{-}}=\sqrt{2} \Pi_{1}^{(2)}(s, s, b), \\
& \Pi_{b}^{*-} \rightarrow \Xi_{b}^{0} K^{-}=\Pi_{1}^{(2)}(u, s, b), \\
& \Pi_{b}^{*-} \rightarrow \Xi_{b}^{-} \eta_{1}=\frac{1}{\sqrt{6}}\left[\Pi_{1}^{(2)}(d, s, b)+2 \Pi_{1}^{(2)}(s, d, b)\right],
\end{aligned}
$$

In the case of charmed baryons it is enough to make the replacement $b \rightarrow c$ and increas the charge of each baryon by a positive unit.

\section{References}

[1] P. Biassoni, arXiv:1009.2627 (2010).

[2] G. Kane, (ed.), and A. Pierce, (ed.), "Perspectives on LHC physics", (Michigan U.). 2008. 337pp. Hackensack, USA: World Scientific (2008) 337 p.

[3] M. Artuso et al., CLEO Collaboration, Phys. Rev. D 65, 071101 (2002).

[4] R. Ammar et al., CLEO Collaboration, Phys. Rev. Lett. 86, 1167 (2001).

[5] J. M. Link, et al., FOCUS Collaboration, Phys. Lett. B 525, 2005 (2002).

[6] S. B. Athar, et al., CLEO Collaboration, Phys. Rev. D 71, 051101 (2005).

[7] L. Gibbons et al., CLEO Collaboration, Phys. Rev. Lett. 77, 810 (1996).

[8] P. Avery et al., CLEO Collaboration, Phys. Rev. Lett. 75, 4364 (1995).

[9] M. A. Shifman, A. I. Vainshtein and V. I. Zakharov, Nucl. Phys. B 147, 385 (1979).

[10] V. M. Braun, prep: hep-ph/9801222 (1998).

[11] T. M. Aliev, K. Azizi, M. Savc1, arXiv: hep-ph/1011.0086 (2010).

[12] T. M. Aliev, K. Azizi, M. Savc1, arXiv: hep-ph/1009.3658 (2010). 
[13] V. M. Belyaev and B. L. Ioffe, Sov. Phys. JETP, 57, 716 (1982).

[14] T. M. Aliev, A. Özpineci, S. B. Yakovlev, V. Zamiralov, Phys. Rev. D 74, 116001 (2006).

[15] T. M. Aliev, A. Özpineci, M. Savcı and V. Zamiralov, Phys. Rev. D 80, 016010 (2009).

[16] T. M. Aliev, K. Azizi, A. Özpineci and M. Savcı, Phys. Rev. D 80, 096003 (2009).

[17] T. M. Aliev, A. Özpineci, M. Savcı and V. Zamiralov, Phys. Rev. D 81, 056004 (2010).

[18] E. Bagan, M. Chabab, H. G. Dosch and S. Narison, Phys. Lett. B 278, 369 (1992).

[19] P. Ball, V. M. Braun, and A. Lenz, JHEP 065, 004 (2006).

[20] I. I. Balitsky and V. M. Braun, Nucl. Phys. B 311, 541 (1989).

[21] K. G. Chetyrkin, A. Khodjamirian, and A. A. Pivovarov, Phys. Lett. B 651, 250 (2008).

[22] I. I. Balitsky, V. M. Braun, and A. V. Kolesnichenko, Nucl. Phys. B 312, 509 (1989).

[23] T. M. Aliev, K. Azizi, A. Özpineci, Phys. Rev. D 79, 056005 (2009).

[24] E. Bagan, M. Chabab, H. G. Dosch and S. Narison, Phys. Lett. B 287, 176 (1992).

[25] S. Tawfig, P. J. O’Donnell, J. G. Korner, Phys. Rev. D 58, 054010 (1998).

[26] J. L. Rosner, Phys. Rev. D 52, 6461 (1995).

[27] M. A. Ivanov, J. G. Korner, V. E. Lyubovitskij, A. G. Rusetsky, Phys. Rev. D 60, 094002 (1999).

[28] C. Albertus, E. Hernandez, J. Nieves and J. M. Verde-Velasco, Phys. Rev. D 72 , 094022 (2005).

[29] K. Nakamura et al., Particle Data Group, J. Phys. G 37, 075021 (2010). 